\title{
Early-onset sepsis in term infants admitted to neonatal intensive care units (2011-2016)
}

\author{
Laura Bech Polcwiartek ${ }^{1,2} \cdot$ P. Brian Smith ${ }^{1,3}$. Daniel K. Benjamin ${ }^{4} \cdot K_{\text {Kanecia Zimmerman }}^{1,3} \cdot$ Alycia Love $^{1}$. \\ Lance Tiu ${ }^{1} \cdot$ Sylvia Murray ${ }^{1} \cdot$ Phillip Kang ${ }^{1} \cdot$ Finn Ebbesen ${ }^{2} \cdot$ Søren Hagstrøm $\mathbb{D}^{2,5} \cdot$ Reese H. Clark $\mathbb{D}^{6}$. \\ Rachel G. Greenberg $\mathbb{1}^{1,3}$
}

Received: 5 May 2020 / Revised: 8 September 2020 / Accepted: 6 October 2020 / Published online: 17 October 2020

(c) The Author(s), under exclusive licence to Springer Nature America, Inc. 2020

\begin{abstract}
Objectives Investigate characteristics of term infants culture-evaluated for early-onset sepsis (EOS) in neonatal intensive care units (NICUs), frequencies of organisms causing EOS, and factors associated with EOS.

Study design Using a cohort design, we identified term infants evaluated for EOS with blood, cerebrospinal fluid, or urine cultures in 326 NICUs (2011-2016). Using multivariable logistic regression, we investigated the association between EOS and demographic characteristics.

Results Of 142,410 infants, 1197 (0.8\%) had EOS, most commonly caused by group B Streptococcus (GBS; 40.6\%). Lower EOS risk was associated with low Apgar score, Cesarean delivery, small for gestational age, prenatal antibiotic exposure, and positive or unknown maternal GBS screening result. Increased risk was associated with prolonged rupture of membranes, maternal age $<19$ years, vasopressor treatment, and ventilator support.

Conclusion(s) GBS was the most frequent cause of EOS. Early risk factor recognition may help daily management of term infants in NICUs.
\end{abstract}

\section{Introduction}

Sepsis is a leading cause of neonatal morbidity and mortality worldwide. Estimates indicate that sepsis within the first postnatal month accounts for $\sim 2000$ deaths each year in the United States and, among these deaths, the majority occur within the first postnatal week [1]. Sepsis within the

Rachel G. Greenberg

rachel.greenberg@duke.edu

1 Duke Clinical Research Institute, Duke University, Durham, NC, USA

2 Department of Pediatrics, Aalborg University Hospital, Aalborg, Denmark

3 Department of Pediatrics, Duke University School of Medicine, Durham, NC, USA

4 Department of Economics, Clemson University, Clemson, SC, USA

5 Department of Clinical Medicine, Aalborg University, Aalborg, Denmark

6 Pediatrix Medical Group, Sunrise, FL, USA first three postnatal days is defined as early-onset sepsis (EOS). Most frequently, EOS is caused by vertical transmission, and despite implementation of guidelines on screening for maternal group B Streptococcus (GBS) colonization, GBS continues to be the most frequent organism causing EOS [2-4].

In many cases, infants with EOS appear to be well or present with nonspecific signs often associated with normal transition to extrauterine life [2]. This vague presentation often challenges the decision on which infants should be treated with empiric antibiotics prior to the availability of culture results [5]. Limiting the use of empiric antibiotics in uninfected infants is important, because excessive use of antibiotics may lead to emergence of resistant flora and changes in the microbiome [2]. Furthermore, separation of hospitalized infants and parents may be associated with infant and family discord and stress [6]. Therefore, early identification of infants at risk of EOS is clinically important and may potentially reduce mortality and long-term morbidities [7].

Previous studies have developed a predictive sepsis risk model based on the combination of infants' clinical presentation and the following factors: gestational age (GA), highest intrapartum temperature, duration of membrane 
rupture, intrapartum antibiotic treatment, and maternal GBS status [2, 8]. Recently, the models have been evaluated with positive results showing that their implementation may reduce empiric antibiotic use $[5,7,9,10]$. Such changes in clinical practice may alter the epidemiology of EOS, making continued surveillance within this field warranted $[11,12]$. Such surveillance would help clinicians in making rational antibiotic choices and provide optimal treatment strategies in infants with EOS [4].

Therefore, through a large cohort of infants discharged from neonatal intensive care units (NICUs), we sought to investigate the epidemiology of EOS in term infants. Specifically, we investigated: (1) characteristics of infants evaluated for EOS with cultures; (2) the frequencies of organisms causing EOS; and (3) factors associated with EOS.

\section{Methods}

\section{Study design and population}

Using a cohort study design, we identified all infants discharged from a NICU during a 6-year period from 2011 to 2016 in a database managed by the Pediatrix Medical Group. We chose the study period because guidelines on screening for maternal GBS colonization were last revised in 2010. From this group of infants, we included only inborn term ( $\geq 37$ to $\leq 42$ gestational weeks at birth) infants with a blood, cerebrospinal fluid (CSF), or urine culture drawn within the first 3 postnatal days. We excluded infants with major congenital anomalies.

\section{Data and variables}

The Pediatrix Medical Group Clinical Data Warehouse prospectively captures clinical information entered into an electronic health record by clinicians at 392 NICUs in the United States. Data on multiple aspects of care are entered into the system to generate admission notes, daily progress notes, procedure notes, and discharge summaries. We obtained data on GA at time of birth; birth weight; small for gestational age (SGA); sex; race and ethnicity; Apgar score at 5 min; maternal age; delivery type; maternal GBS screening results; prolonged rupture of membranes (PROM); prenatal antibiotic exposure; prenatal steroid exposure; age at evaluation with cultures from blood, CSF, or urine; type of organism causing EOS; age at initiation of ventilator support; age at initiation of vasopressor treatment; mortality; and length of hospitalization.

\section{Definitions}

We defined major anomaly as an anomaly present at birth that was lethal, life-shortening, life-threatening, requiring major surgery, or affecting the infant's quality of life in a significant way. We defined ventilator support prior to culture as initiation of mechanical ventilation (conventional mechanical ventilation or high-frequency ventilation) on or before the day of the initial culture. We defined vasopressor treatment prior to culture as initiation of one of the following drugs on or before the day of the initial culture: dobutamine, dopamine, epinephrine, milrinone, norepinephrine, or vasopressin.

\section{Outcome measures}

Our primary outcome was EOS, which was defined as a culture positive for a bacterial or fungal organism obtained from blood, CSF, or urine within the first 3 postnatal days. Urine cultures included samples collected from in-and-out catheters or suprapubic taps. We excluded cultures for organisms normally considered contaminants such as nonspeciated streptococci, Bacillus sp., Corynebacterium sp., diphtheroids sp., Gram-positive rods (not including Listeria sp.), Lactobacillus sp., Micrococcus sp., Stomatococcus sp., and Bacteroides sp. We included positive cultures for coagulase-negative Staphylococcus if two positive cultures were identified within a 4-day period [13]. We considered multiple positive cultures of a single organism on different days to be a single episode of EOS. However, in the analysis of organism frequency, cultures positive for different organism types were included once per organism type; consequently, in reporting of organism frequency, some infants were included more than once.

\section{Statistical analysis}

Categorical variables were reported as counts with corresponding percentages and continuous variables as medians with 25 th and 75 th percentiles. We reported the frequency of EOS and patient characteristics. Among infants with EOS, we reported the frequency of organisms causing EOS according to culture type.

We investigated the association between predetermined factors and EOS in a multivariable logistic regression model and calculated odds ratios (ORs) with corresponding 95\% confidence intervals (CIs). Factors included were: GA at time of birth, SGA, sex, race, and ethnicity, Apgar score groups (0-3, 4-6, 7-10) at $5 \mathrm{~min}$, maternal age, delivery type, maternal GBS screening results, PROM, prenatal care status, prenatal antibiotic exposure, prenatal steroid exposure, and pre-culture ventilator support, and vasopressor treatment. In the regression analysis, infants with multiple positive cultures were considered as a single episode of EOS.

A two-sided $p$ value $<0.05$ was considered statistically significant. Data management and statistical analyses were performed using Stata, version 16.1 (StataCorp, College Station, Texas, USA). This project received Duke University 
Fig. 1 Inclusion and exclusion of infants discharged from a NICU. Flow chart of inclusion and exclusion of infants discharged from a NICU evaluated for EOS. CSF cerebrospinal fluid, EOS earlyonset sepsis, GA gestational age, NICU neonatal intensive care unit.

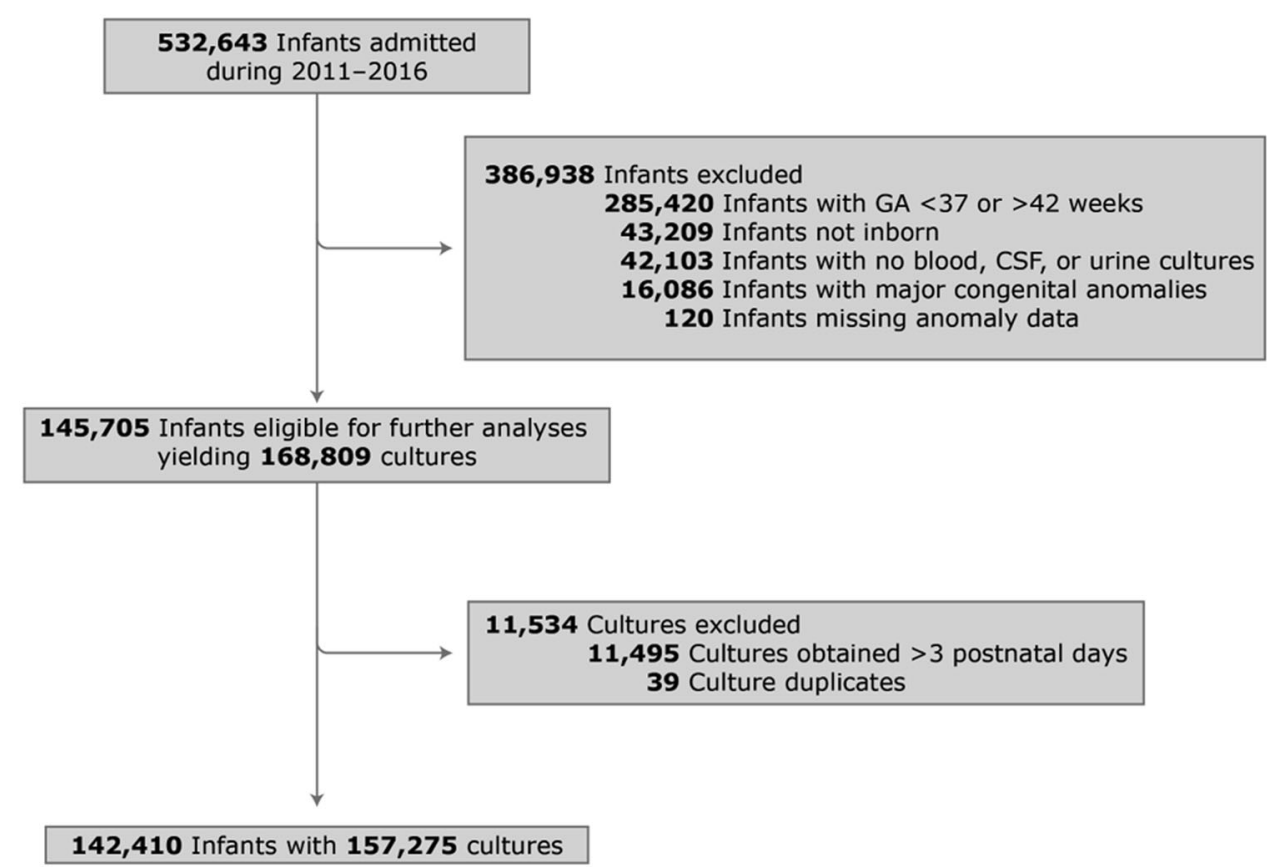

Institutional Review Board approval with a waiver of informed consent.

\section{Results}

\section{Patients and characteristics}

During the study period, 142,410 term infants had a culture obtained within 3 postnatal days. Of these, $1197(0.8 \%)$ had EOS (Fig. 1). Infants were most commonly male and white, the median GA at time of birth was 39 weeks $(38,40)$ and the median birth weight was $3348 \mathrm{~g}(3000,3708)$. Full sample mortality was $0.2 \%$, and $0.8 \%$ and $0.2 \%$ among infants with and without EOS, respectively (Table 1). Among those who received mechanical ventilation on or before the culture day, the median duration was $3(2,5)$ days and $2(1,3)$ days among infants with and without EOS, respectively. Among those who received vasopressor treatment on or before the culture day, the median duration was $3(2,5)$ days and $2(1,4)$ days among infants with and without EOS, respectively. Compared to infants without EOS, a higher percentage of infants with EOS received mechanical ventilation $(10.9 \%$ vs. $6.7 \%)$ or continuous positive airway pressure ( $32 \%$ vs. $28 \%$ ) at some point during the entire hospitalization. Among infants who were discharged home, the median length of hospital stay was 6 $(4,8)$ days overall and $11(8,14)$ and $6(4,8)$ for those with and without EOS, respectively.

\section{Frequency of pathogens}

Among the 1197 infants with EOS, 1236 organisms were isolated from blood $(95.3 \%, 1178 / 1236)$ or CSF $(4.7 \%$, 58/1236). No urine cultures met the inclusion criteria. Overall, GBS was the most frequent pathogen $(40.6 \%$, 502/1236) followed by Escherichia coli (E. coli) (12.3\%, 152/1236) and Enterococcus species. (5.1\%, 63/1236). GBS was isolated from $40.2 \%$ (473/1178) of all blood cultures, and from $50.0 \%$ (29/58) of all CSF cultures (Table 2).

\section{Factors associated with risk of EOS}

On multivariable regression analysis, neither sex nor race was associated with risk of EOS. Low Apgar score (OR $0.37,95 \%$ CI $0.20-0.69$ ), cesarean delivery (OR $0.66,95 \%$ CI 0.57-0.76), SGA (OR 0.70, 95\% CI 0.54-0.90), prenatal antibiotic exposure (OR $0.70,95 \%$ CI $0.61-0.81$ ), positive GBS screening result (OR 0.83, 95\% CI 0.70-0.99), missing/unknown GBS result (OR 0.73, 95\% CI 0.58-0.92), vasopressor exposure on or before the culture day (OR 3.64, 95\% CI 2.51-5.29), and ventilator exposure on or before the culture day (OR 1.58, 95\% CI 1.19-2.10) were associated with lower risk of EOS. Infants of mothers with PROM (OR $1.71,95 \%$ CI 1.42-2.05) had increased risk of EOS. In addition, compared to maternal age 20-29 years, maternal age $\leq 19$ years was associated with increased risk of EOS (OR 1.35, 95\% CI 1.13-1.62) (Table 3). 
Table 1 Characteristics of the study population.

\begin{tabular}{|c|c|c|c|}
\hline & $\begin{array}{l}\text { Infants without EOS } \\
(n=141,213)\end{array}$ & $\begin{array}{l}\text { Infants with EOS } \\
(n=1197)\end{array}$ & $\begin{array}{l}\text { Total study population } \\
(n=142,410)\end{array}$ \\
\hline Gestational age, weeks & $39[38,40]$ & $39[38,40]$ & $39[38,40]$ \\
\hline Birth weight, $g$ & $3347[3000,3709]$ & $3379[3047,3660]$ & $3348[3000,3708]$ \\
\hline Male & $57.8 \%$ & $55.8 \%$ & $57.8 \%$ \\
\hline \multicolumn{4}{|l|}{ Race/ethnicity } \\
\hline White & $47.6 \%$ & $45.2 \%$ & $47.6 \%$ \\
\hline Black & $16.8 \%$ & $15.8 \%$ & $16.8 \%$ \\
\hline Hispanic & $28.4 \%$ & $31.2 \%$ & $28.5 \%$ \\
\hline Other & $7.2 \%$ & $7.8 \%$ & $7.2 \%$ \\
\hline \multicolumn{4}{|l|}{ Apgar score at $5 \mathrm{~min}$} \\
\hline $0-3$ & $2.0 \%$ & $1.5 \%$ & $2.0 \%$ \\
\hline $4-6$ & $6.6 \%$ & $6.6 \%$ & $6.6 \%$ \\
\hline $7-10$ & $91.4 \%$ & $91.8 \%$ & $91.4 \%$ \\
\hline Maternal age at birth, years & $28[23,32]$ & $27[22,31]$ & $28[23,32]$ \\
\hline Cesarean section & $47.3 \%$ & $35.4 \%$ & $47.2 \%$ \\
\hline $\begin{array}{l}\text { Positive maternal GBS } \\
\text { colonization status }\end{array}$ & $22.2 \%$ & $17.4 \%$ & $22.1 \%$ \\
\hline Prolonged rupture of membranes & $9.3 \%$ & $14.5 \%$ & $9.3 \%$ \\
\hline Prenatal antibiotic exposure & $41.1 \%$ & $32.0 \%$ & $41.1 \%$ \\
\hline Prenatal steroid exposure & $1.4 \%$ & $1.5 \%$ & $1.4 \%$ \\
\hline $\begin{array}{l}\text { Ventilator on or before day of } \\
\text { culture }\end{array}$ & $5.6 \%$ & $8.9 \%$ & $5.6 \%$ \\
\hline $\begin{array}{l}\text { Vasopressor treatment on or } \\
\text { before day of culture }\end{array}$ & $0.9 \%$ & $3.3 \%$ & $0.9 \%$ \\
\hline Died & $0.2 \%$ & $0.8 \%$ & $0.2 \%$ \\
\hline \multicolumn{4}{|l|}{ Discharge year } \\
\hline 2011 & $15.8 \%$ & $17.4 \%$ & $15.8 \%$ \\
\hline 2012 & $16.1 \%$ & $15.7 \%$ & $16.1 \%$ \\
\hline 2013 & $16.3 \%$ & $16.0 \%$ & $16.3 \%$ \\
\hline 2014 & $17.6 \%$ & $17.0 \%$ & $17.6 \%$ \\
\hline 2015 & $17.6 \%$ & $16.4 \%$ & $17.5 \%$ \\
\hline 2016 & $16.7 \%$ & $17.5 \%$ & $16.7 \%$ \\
\hline
\end{tabular}

Values presented as \% or median [p25-p75].

EOS early-onset sepsis, GBS group B Streptococcus, NIPPV noninvasive positive pressure ventilation, $C P A P$ continuous positive airway pressure, $N C$ nasal cannula.

\section{Discussion}

In this cohort study, we identified 142,410 term infants who were evaluated for EOS in a NICU during 2011-2016. EOS occurred in $0.8 \%$ (1197), and was most often caused by GBS (40.6\%), E. coli (12.3\%), or Enterococcus (5.1\%). Low Apgar score, cesarean delivery, SGA, and prenatal antibiotic exposure were associated with decreased risk of EOS, while PROM, negative GBS screening result, and low maternal age conferred increased risk.

Recent studies reported EOS incidences of 0.7, 0.5, 1.2, and 1.1 per 1000 live births from 2005 to 2014,2015 to 2016, 2002 to 2012, and 2015 to 2017, respectively $[9,11,14,15]$. We found an incidence of 8.4 per 1000 infants evaluated for EOS, but because our sample was drawn solely from infants evaluated for EOS with cultures and discharged from a NICU, the incidence of EOS found in our study is not comparable with the other studies. Nevertheless, studies generally agree that the incidence of EOS has decreased after guidelines on screening for maternal GBS colonization were issued in 2002 and revised in 2010 [5, 15-17]. Interestingly, some studies suggest that the use of empiric antibiotic treatment has not decreased along with the decreasing incidence of EOS, leading to concerns about emerging resistant flora and antibiotic exposure of a large number of uninfected infants [5, 18]. Recent findings of an association between early antibiotic treatment and subsequent childhood morbidity such as 
Table 2 Organism causing EOS.

\begin{tabular}{|c|c|c|c|}
\hline \multirow[b]{2}{*}{ Organisms } & \multicolumn{3}{|l|}{ Culture type } \\
\hline & $\begin{array}{l}\text { All } \\
(n=1236)\end{array}$ & $\begin{array}{l}\text { Blood } \\
(n=1178)\end{array}$ & $\begin{array}{l}\text { Cerebrospinal } \\
\text { fluid }(n=58)\end{array}$ \\
\hline \multicolumn{4}{|l|}{ Gram positive } \\
\hline $\begin{array}{c}\text { Group B } \\
\text { Streptococcus }\end{array}$ & $502(40.6)$ & $473(40.2)$ & $29(50.0)$ \\
\hline $\begin{array}{l}\text { Gram-positive } \\
\text { cocci (unspecified) }\end{array}$ & $212(17.2)$ & $208(17.7)$ & $4(6.9)$ \\
\hline Enterococcus spp. & $63(5.1)$ & $63(5.4)$ & $0(0.0)$ \\
\hline $\begin{array}{l}\text { Staphylococcus } \\
\text { aureus }\end{array}$ & $23(1.9)$ & $23(2.0)$ & $0(0.0)$ \\
\hline $\begin{array}{l}\text { Coagulase- } \\
\text { negative } \\
\text { Staphylococcus }\end{array}$ & $20(1.6)$ & $18(1.5)$ & $2(3.5)$ \\
\hline $\begin{array}{l}\text { Streptococcus } \\
\text { pneumoniae }\end{array}$ & $11(0.9)$ & $11(0.9)$ & $0(0.0)$ \\
\hline Listeria spp. & $4(0.3)$ & $4(0.3)$ & $0(0.0)$ \\
\hline $\begin{array}{c}\text { Group A } \\
\text { Streptococcus }\end{array}$ & $2(0.2)$ & $1(0.1)$ & $1(1.7)$ \\
\hline \multicolumn{4}{|l|}{ Gram negative } \\
\hline Escherichia coli & $152(12.3)$ & $145(12.3)$ & $7(12.1)$ \\
\hline $\begin{array}{l}\text { Gram-negative } \\
\text { rods (unspecified) }\end{array}$ & $39(3.2)$ & $37(3.1)$ & $2(3.5)$ \\
\hline $\begin{array}{l}\text { Haemophilus } \\
\text { influenza }\end{array}$ & $23(1.9)$ & $23(2.0)$ & $0(0.0)$ \\
\hline Klebsiella spp. & $7(0.6)$ & $7(0.6)$ & $0(0.0)$ \\
\hline Acinetobacter spp. & $6(0.5)$ & $4(0.3)$ & $2(3.5)$ \\
\hline Enterobacter spp. & $4(0.3)$ & $4(0.3)$ & $0(0.0)$ \\
\hline Citrobacter spp. & $3(0.2)$ & $3(0.3)$ & $0(0.0)$ \\
\hline Pseudomonas spp. & $3(0.2)$ & $2(0.2)$ & $1(1.7)$ \\
\hline Salmonella spp. & $1(0.1)$ & $1(0.1)$ & $0(0.0)$ \\
\hline Serratia spp. & $1(0.1)$ & $1(0.8)$ & $0(0.0)$ \\
\hline \multicolumn{4}{|l|}{ Fungus } \\
\hline Candida spp. & $1(0.1)$ & $1(0.1)$ & $0(0.0)$ \\
\hline Unknown & $159(12.9)$ & $149(12.7)$ & $10(17.2)$ \\
\hline
\end{tabular}

Values presented as no. (\%) or median [p25-p75].

EOS early-onset sepsis, spp. species.

asthma and obesity have underscored the importance of limiting unnecessary antibiotic treatment and, when indicated, making rational empiric antibiotic decisions [2, 5]. Therefore, a better understanding of the changing epidemiology of EOS is needed.

Previous studies investigating trends in organisms causing EOS have reported a shift in the distribution of organisms causing EOS toward a predominance of Gram-negative rods, especially E. coli $[12,19]$. Lin et al. reported that E.coli caused $40.9 \%$ of EOS episodes from 2001 to 2004 and $70 \%$ from 2005 to 2008 [19]. The same trends were reported in a study by Sgro et al. during the same study period [12]. In contrast, we found GBS to be the most frequent known
Table 3 Association between factors and risk of EOS on multivariable logistic regression analysis.

\begin{tabular}{|c|c|c|c|}
\hline Variable & Adjusted OR & $95 \% \mathrm{CI}$ & $p$ value \\
\hline \multicolumn{4}{|l|}{$\begin{array}{l}\text { Gestational age (reference: } \\
40 \text { weeks) }\end{array}$} \\
\hline 37 & 0.89 & $(0.71-1.10)$ & 0.28 \\
\hline 38 & 1.09 & $(0.92-1.31)$ & 0.32 \\
\hline 39 & 0.95 & $(0.81-1.13)$ & 0.59 \\
\hline 41 & 1.11 & $(0.83-1.47)$ & 0.49 \\
\hline Small for gestational age & 0.70 & $(0.54-0.90)$ & 0.006 \\
\hline Male & 0.98 & $(0.87-1.10)$ & 0.70 \\
\hline \multicolumn{4}{|l|}{$\begin{array}{l}\text { Race/ethnicity } \\
\text { (reference: White) }\end{array}$} \\
\hline Black & 1.05 & $(0.86-1.28)$ & 0.67 \\
\hline Hispanic & 1.13 & $(0.97-1.32)$ & 0.11 \\
\hline Other & 1.15 & $(0.91-1.47)$ & 0.24 \\
\hline \multicolumn{4}{|l|}{$\begin{array}{l}\text { Apgar score at } 5 \text { min } \\
\text { (reference: } 7-10 \text { ) }\end{array}$} \\
\hline $0-3$ & 0.37 & $(0.20-0.69)$ & 0.002 \\
\hline $4-6$ & 0.81 & $(0.61-1.07)$ & 0.13 \\
\hline \multicolumn{4}{|l|}{$\begin{array}{l}\text { Maternal age at birth } \\
\text { (reference: 20-29) }\end{array}$} \\
\hline$\leq 19$ years & 1.35 & $(1.13-1.62)$ & 0.001 \\
\hline 30-39 years & 0.87 & $(0.74-1.01)$ & 0.06 \\
\hline$\geq 40$ years & 0.72 & $(0.46-1.13)$ & 0.15 \\
\hline Cesarean section & 0.66 & $(0.57-0.76)$ & $<0.001$ \\
\hline \multicolumn{4}{|l|}{$\begin{array}{l}\text { GBS colonization status } \\
\text { (reference: negative) }\end{array}$} \\
\hline Positive & 0.83 & $(0.70-0.99)$ & 0.04 \\
\hline Missing or unknown & 0.73 & $(0.58-0.92)$ & 0.008 \\
\hline $\begin{array}{l}\text { Prolonged rupture of the } \\
\text { membranes }\end{array}$ & 1.71 & $(1.42-2.05)$ & $<0.001$ \\
\hline Prenatal care & 0.90 & $(0.69-1.18)$ & 0.45 \\
\hline Prenatal antibiotic exposure & 0.70 & $(0.61-0.81)$ & $<0.001$ \\
\hline Prenatal steroid exposure & 1.10 & $(0.66-1.82)$ & 0.72 \\
\hline $\begin{array}{l}\text { Vasopressor treatment on or } \\
\text { before day of culture }\end{array}$ & 3.64 & $(2.51-5.29)$ & $<0.001$ \\
\hline $\begin{array}{l}\text { Ventilator on or before day of } \\
\text { culture }\end{array}$ & 1.58 & $(1.19-2.10)$ & 0.001 \\
\hline \multicolumn{4}{|l|}{$\begin{array}{l}\text { Discharge year } \\
\text { (reference: 2011) }\end{array}$} \\
\hline 2012 & 0.88 & $(0.71-1.11)$ & 0.28 \\
\hline 2013 & 0.92 & $(0.74-1.14)$ & 0.45 \\
\hline 2014 & 0.90 & $(0.71-1.13)$ & 0.35 \\
\hline 2015 & 0.86 & $(0.70-1.05)$ & 0.14 \\
\hline 2016 & 0.98 & $(0.79-1.21)$ & 0.85 \\
\hline
\end{tabular}

CI confidence interval, EOS early-onset sepsis, GBS group B Streptococcus, OR odds ratio.

organism, suggesting that clinicians should consider choosing empiric antibiotic treatment targeting GBS. Comparative studies within this field are limited by different and relatively 
short study periods, varying study populations, and different culture types $[2,11,12,15]$. While we only included term infants, several studies included preterm or extreme preterm infants [11,20,21], which may explain the predominance of Gram-negative rods previously reported [12]. Consistent with our findings, a recent study by Singh et al. reported GBS to be the most prevalent cause of EOS in term infants admitted to the NICU, accounting for $62 \%$ of cases [11]. In comparison, only $40.6 \%$ of infants with EOS had GBS in our study; however, we used data from 2011 to 2016, whereas Singh et al. used data from 2002 to 2012 [11]. We speculate that the observed difference in GBS frequencies observed in our study and in Singh et al.'s study may be a direct consequence of the effect GBS screening has had since 2002. Notably, because our data include a rather large number of unspecified Grampositive cocci, the GBS frequency in our study may be underestimated. It is also possible that some of these unspecified Gram-positive cocci were contaminants, and the frequency of EOS could have been overestimated.

Because the burden of EOS remains substantial despite implementation of maternal GBS screening and targeted use of antibiotics, early identification of EOS remains important $[22,23]$. As a result, in a 2011 study, a predictive sepsis risk model among infants with $\mathrm{GA} \geq 34$ weeks was developed [8]. The purpose of this model was to help clinicians in subsequent decisions on neonatal management and safely reduce the number of infants evaluated for EOS. Recently, the clinical use of this model has been validated in several studies that showed a significant reduction in the use of empiric antibiotic treatment following its implementation $[2,7,9,10]$. Among others studies, Puopolo et al. identified PROM and intrapartum fever as factors associated with risk of EOS, and despite differences in study design, population, and period, we also found PROM to be a risk factor [8]. In contrast, although prior studies have reported that risk of EOS was inversely associated with GA and birth weight [8, 24], we found no association between lower-term GA and EOS, and a decreased risk of EOS among SGA infants. We speculate that our findings may reflect the fact that term infants are often admitted to the NICU due to clinical signs of disease, whereas term SGA infants are more likely to be admitted simply because they are SGA and possibly immature. A similar explanation may also account for the association between low Apgar score and decreased risk of EOS in our study. Infants with an Apgar score of 0-3 are most likely admitted to the NICU because of low Apgar score indicating asphyxia, whereas infants with normal Apgar score are admitted due to signs of disease rather than the Apgar score per se. Importantly, another potential reason for the association between low Apgar score and decreased risk of EOS may also be that low Apgar score can be caused by various conditions such as meconiumstained amniotic fluid, and clinical guidelines regarding management of some of these condition may recommend antibiotic treatment immediately after birth [25]. Nonetheless, an explanation for why we found an inverse association between maternal age and risk of EOS remains unclear, and further work needs to be done to understand this. Cesarean delivery was associated with a lower risk of EOS, because EOS is caused most frequently by vertical transmission. Finally, Hispanic ethnicity has previously been associated with elevated risk of EOS, which raises concerns about delayed or limited access to healthcare and low rates of prenatal care in this particular population [26]. Interestingly, despite having a comparable study cohort to this study, we found no association between race or ethnicity and risk of EOS.

We found that mortality was relatively low in our cohort overall $(0.2 \%)$, and remained low even in infants with EOS $(0.8 \%)$. Our findings are consistent with a recent report by the National Institute of Child Health and Human Development Neonatal Research Network, in which no term infants with EOS died [14]. We used a microbiologic definition of sepsis, which is consistent with previous studies of infants, but which may account for the low mortality compared to sepsis in other populations. For example, it is possible that some infants with microbiologic sepsis were actually uninfected or had transient bacteremia of limited clinical significance. Alternatively, it is possible that EOS in infants simply manifests differently than in other age groups.

Our study had some limitations. First, our database did not contain information on antibiotic resistance, clinical signs and symptoms such as maternal fever or infant organ dysfunction, maternal comorbidities, socioeconomic status, and presence of chorioamnionitis. Second, our data included a rather large proportion of bacteria of unknown type $(12.9 \%)$. Finally, our population included only NICU infants who had an evaluation for EOS, which allowed us to eliminate infants with no clinical suspicion for EOS. Notably, however, our data did not indicate why each culture was obtained, and different clinicians are likely to have various thresholds for ordering cultures.

In conclusion, we identified GBS as the most frequent organism causing EOS in our study of term infants hospitalized in the NICU. Therefore, clinicians should continue to be aware of this organism when treating term infants with putative EOS. In addition, we identified several factors associated with EOS, and early recognition of these risk factors may aid in the daily management of term infants admitted to NICUs.

Acknowledgements PTN Steering Committee Members: Daniel K. Benjamin Jr., Christoph Hornik, Kanecia Zimmerman, Phyllis Kennel, and Rose Beci, Duke Clinical Research Institute, Durham, NC; Chi Dang Hornik, Duke University Medical Center, Durham, NC; Gregory L. Kearns, Scottsdale, AZ; Matthew Laughon, University of North 
Carolina at Chapel Hill, Chapel Hill, NC; Ian M. Paul, Penn State College of Medicine, Hershey, PA; Janice Sullivan, University of Louisville, Louisville, KY; Kelly Wade, Children's Hospital of Philadelphia, Philadelphia, PA; Paula Delmore, Wichita Medical Research and Education Foundation, Wichita, KS. The Eunice Kennedy Shriver National Institute of Child Health and Human Development (NICHD): Perdita Taylor-Zapata and June Lee. The Emmes Company, LLC (Data Coordinating Center): Ravinder Anand, Gaurav Sharma, Gina Simone, Kim Kaneshige, and Lawrence Taylor. PTN Publications Committee: Chaired by Thomas Green, Ann \& Robert H. Lurie Children's Hospital of Chicago, Chicago, IL.

Funding This work was supported by Duke Clinical Research Institute's R25 Summer Training in Academic Research (STAR) Program (Grant \#5R25HD076475-07).

\section{Compliance with ethical standards}

Conflict of interest PBS reported being a member of Data and Safety Monitoring Board for United Therapeutics and Seelos; funding from NIH-U2C-RFA-OD-16-006. KZ serves as a consultant to industry for pediatric drug development. RGG reported receiving support from industry for research services (https://dcri.org/about-us/conflict-ofinterest/). The other authors declare that they have no conflict of interest.

Publisher's note Springer Nature remains neutral with regard to jurisdictional claims in published maps and institutional affiliations.

\section{References}

1. The United Nations Children's Fund (UNICEF). The neonatal period is the most vulnerable time for a child. 2018. https://data.unicef.org/ topic/child-survival/neonatal-mortality/. Accessed 7 Apr 2020.

2. Kuzniewicz MW, Puopolo KM, Fischer A, Walsh EM, Li S, Newman TB, et al. A quantitative, risk-based approach to the management of neonatal early-onset sepsis. JAMA Pediatr. 2017; 171:365-71.

3. Ohlsson A, Shah VS. Intrapartum antibiotics for known maternal Group B streptococcal colonization. Cochrane Database Syst Rev. 2014:CD007467.

4. Stoll BJ, Hansen NI, Sanchez PJ, Faix RG, Poindexter BB, Van Meurs KP, et al. Early onset neonatal sepsis: the burden of group B Streptococcal and E. coli disease continues. Pediatrics. 2011; 127:817-26.

5. Kuzniewicz MW, Walsh EM, Li S, Fischer A, Escobar GJ. Development and implementation of an early-onset sepsis calculator to guide antibiotic management in late preterm and term neonates. Jt Comm J Qual Patient Saf. 2016;42:232-9.

6. Ray JG, Urquia ML, Berger H, Vermeulen MJ. Maternal and neonatal separation and mortality associated with concurrent admissions to intensive care units. CMAJ. 2012;184: E956-62.

7. Warren S, Garcia M, Hankins C. Impact of neonatal early-onset sepsis calculator on antibiotic use within two tertiary healthcare centers. J Perinatol. 2017;37:394-7.

8. Puopolo KM, Draper D, Wi S, Newman TB, Zupancic J, Lieberman E, et al. Estimating the probability of neonatal earlyonset infection on the basis of maternal risk factors. Pediatrics. 2011;128:1155-63.
9. Dhudasia MB, Mukhopadhyay S, Puopolo KM. Implementation of the sepsis risk calculator at an academic birth hospital. Hosp Pediatr. 2018;8:243-50.

10. Beavers JB, Bai S, Perry J, Simpson J, Peeples S. Implementation and evaluation of the early-onset sepsis risk calculator in a highrisk university nursery. Clin Pediatr. 2018;57:1080-5.

11. Singh T, Barnes EH, Isaacs D. Australian Study Group for neonatal infections. Early-onset neonatal infections in Australia and New Zealand, 2002-2012. Arch Dis Child Fetal Neonatal Ed. 2018;104:F248-52.

12. Sgro M, Shah PS, Campbell D, Tenuta A, Shivananda S, Lee SK, et al. Early-onset neonatal sepsis: rate and organism pattern between 2003 and 2008. J Perinatol. 2011;31:794-8.

13. Hornik CP, Fort P, Clark RH, Watt K, Benjamin DK Jr, Smith PB, et al. Early and late onset sepsis in very-low-birth-weight infants from a large group of neonatal intensive care units. Early Hum Dev. 2012;88:S69-74.

14. Stoll BJ, Puopolo KM, Hansen NI, Sánchez PJ, Bell EF, Carlo WA, et al. Early-onset neonatal sepsis 2015 to 2017, the rise of Escherichia coli, and the need for novel prevention strategies. JAMA Pediatr. 2020;174:e200593.

15. Cailes B, Kortsalioudaki C, Buttery J, Pattnayak S, Greenough A, Matthes $J$, et al. Epidemiology of UK neonatal infections: the neonIN infection surveillance network. Arch Dis Child Fetal Neonatal Ed. 2018;103:547-53.

16. Brown AP, Denison FC. Selective or universal screening for GBS in pregnancy (review). Early Hum Dev. 2018;126:18-22.

17. Escobar GJ, Puopolo KM, Wi S, Turk BJ, Kuzniewicz MW, Walsh EM, et al. Stratification of risk of early-onset sepsis in newborns $\geq 34$ weeks' gestation. Pediatrics. 2014;133:30-6.

18. Mukhopadhyay S, Eichenwald EC, Puopolo KM. Neonatal earlyonset sepsis evaluations among well-appearing infants: projected impact of changes in CDC GBS guidelines. J Perinatol. 2013; 33:198-205.

19. Lin CY, Hsu CH, Huang FY, Chang JH, Hung HY, Kao HA, et al. The changing face of early-onset neonatal sepsis after the implementation of a maternal group B Streptococcus screening and intrapartum prophylaxis policy-a study in one medical center. Pediatr Neonatol. 2011;52:78-84.

20. Schrag SJ, Farley MM, Petit S, Reingold A, Weston EJ, Pondo T, et al. Epidemiology of invasive early-onset neonatal sepsis, 2005 to 2014. Pediatrics. 2016;138:e20162013. https://doi.org/10.1542/ peds.2016-2013.

21. Nielsen M, Sheikh N, Fitzgerald E, Meehan M, LeBlanc D, Eogan $\mathrm{M}$, et al. Screening for early-onset invasive group B Streptococcal disease in neonates in an Irish hospital (2001-2014): a retrospective audit. Infect Dis. 2017;49:466-70.

22. Weston EJ, Pondo T, Lewis MM, Martell-Cleary P, Morin C, Jewell $\mathrm{B}$, et al. The burden of invasive early-onset neonatal sepsis in the United States, 2005-2008. Pediatr Infect Dis J. 2011;30:937-41.

23. Van Dyke MK, Phares CR, Lynfield R, Thomas AR, Arnold KE, Craig AS, et al. Evaluation of universal antenatal screening for group B streptococcus. N Engl J Med. 2009;360:2626-36.

24. Bandyopadhyay T, Kumar A, Saili A, Randhawa VS. Distribution, antimicrobial resistance and predictors of mortality in neonatal sepsis. J Neonatal Perinat Med. 2018;11:145-53.

25. Garcia-Prats JA. Prevention and management of meconium aspiration syndrome. UpToDate ; 2019. https://www.uptodate. $\mathrm{com} /$ contents/prevention-and-management-of-meconium-aspira tion-syndrome. Accessed 7 Apr 2020.

26. Cohen-Wolkowiez M, Moran C, Benjamin DK, Cotten CM, Clark $\mathrm{RH}$, Benjamin DK Jr., et al. Early and late onset sepsis in late preterm infants. Pediatr Infect Dis J. 2009;28:1052-6. 